\title{
Sternal elevation by the crane technique during pectus excavatum repair: A quantitative analysis
}

\author{
Erik R. de Loos, MD, ${ }^{\mathrm{a}}$ Jean H. T. Daemen, MD, ${ }^{\mathrm{a}, \mathrm{b}}$ Nadine A. Coorens, MSc, ${ }^{\mathrm{a}}$ Jos G. Maessen, MD, PhD, ${ }^{\mathrm{c}, \mathrm{d}}$ \\ Yvonne L. J. Vissers, MD, PhD, ${ }^{a}$ and Karel W. E. Hulsewé, MD, $\mathrm{PhD}^{\mathrm{a}}$
}

\begin{abstract}
Introduction: The crane technique is used to facilitate sternal elevation to provide safe mediastinal passage during the Nuss procedure. The aim was to objectively quantitate the elevation of the crane by 3 -dimensional chest images acquired during the Nuss procedure.
\end{abstract}

Methods: A prospective cohort study was conducted. Patients undergoing the Nuss procedure were eligible. Sternal elevation was achieved by the crane technique providing a simultaneous lift of the anterior chest wall and reduction of the pectus excavatum depth. Both effects were evaluated. Three-dimensional surface images were acquired before incision, following sternal lift, and after bar implantation and quantitatively compared. Reduction of the external pectus excavatum depth was expressed as a percentage.

Results: Thirty patients were included. Ninety percent were male, with a median age of 15.5 years (interquartile range [IQR], 14.5-17.4), Haller index of 3.56 (IQR, 3.09-4.65), and external pectus depth of $18 \mathrm{~mm}$ (IQR, 11-23). Sternal elevation by the crane provided a median $78 \%$ (IQR, 63-100) reduction of the deformity, corresponding with a residual depth of $3 \mathrm{~mm}$ (IQR, $0-7)$. The percentual reduction diminished with increasing depth of the sternal depression (correlation, -0.86). Besides reducing the deformity, the crane caused an elevation of the anterior chest over a large surface area with a maximum lift of $26 \mathrm{~mm}$ (IQR, 19-32).

Conclusions: The crane is an effective sternal elevation technique, providing $78 \%$ reduction of the sternal depression, although its effect lessens with increasing depth. In addition, it produces an elevation of the anterior chest over a large surface area. (JTCVS Techniques 2021;9:167-75)



Intraoperative view of sternal elevation by the crane technique.

\section{CENTRAL MESSAGE}

The crane is an effective sternal

elevation technique, providing

$78 \%$ reduction of the sternal

depression, although its effect

lessens with increasing depth.

\section{PERSPECTIVE}

We have shown that the crane is an effective sternal elevation technique. This expansion of the retrosternal space may facilitate safe mediastinal passage, improve thoracoscopic exposure, diminish risk for scraping of the intrathoracic anterior chest wall where the internal mammary arteries lie, and reduce rotational forces during flipping of the Nuss bar that may prevent intercostal muscle stripping.

See Commentaries on pages 176 and 178
Video clip is available online.

\footnotetext{
From the a Division of General Thoracic Surgery, Department of Surgery, Zuyderland Medical Center, Heerlen; ${ }^{\mathrm{b}}$ Faculty of Health, Medicine and Life Sciences (FHML), School for Oncology and Developmental Biology (GROW), Maastricht; ${ }^{\mathrm{c} D e p a r t-}$ ment of Cardiothoracic Surgery, Maastricht University Medical Center, Maastricht; and ${ }^{\mathrm{d}}$ Faculty of Health, Medicine and Life Sciences (FHML), Cardiovascular Research Institute Maastricht (CARIM), Maastricht, The Netherlands.

This work was supported by the Zuyderland Research and Innovation Fund of Zuyderland Medical Center (Heerlen, The Netherlands) [2019-005].
}

Pectus excavatum is the most common congenital anterior chest wall deformity, with an incidence of 1 in 400 newborns. ${ }^{1,2}$ Symptoms encompass cosmetic discomfort with associated psychosocial burden and physiologic symptoms

Received for publication Feb 1, 2021; accepted for publication May 25, 2021; available ahead of print July 17, 2021.

Address for reprints: Erik R. de Loos, MD, Division of General Thoracic Surgery, Department of Surgery, Zuyderland Medical Center, Henri Dunantstraat 5, 6419PC, Heerlen, The Netherlands (E-mail: e.deloos@zuyderland.nl). 2666-2507

Copyright (C) 2021 The Author(s). Published by Elsevier Inc. on behalf of The American Association for Thoracic Surgery. This is an open access article under the CC BY-NC-ND license (http://creativecommons.org/licenses/by-nc-nd/4.0/). https://doi.org/10.1016/j.xjtc.2021.05.028 


\section{Abbreviations and Acronyms \\ $3 \mathrm{D}=3$-dimensional \\ $\mathrm{BMI}=$ body mass index \\ $\mathrm{IQR}=$ interquartile range}

originating from cardiopulmonary impairment. The latter is caused by the inwardly deviated sternum, compressing the heart and predominantly affecting right ventricular function. ${ }^{3-6}$ The current surgical treatment of choice is the Nuss procedure. It is preferred over the conventional modified Ravitch procedure because it is a less-invasive procedure. ${ }^{7}$ In the Nuss procedure, a substernal bar is implanted to correct the deformity. ${ }^{8}$ Despite being considered minimally invasive, the retrosternal dissection required to pass the Nuss bar through the mediastinum is a potentially hazardous maneuver. In patients with severe pectus excavatum, visualization across the mediastinum may be compromised. In addition, retrosternal passage of the introducer is more difficult and carries greater risk, and cardiac and aortic injuries have been described. ${ }^{9}$ Although these complications are rare, with an estimated incidence of less than $0.1 \%$, when they do occur, they are fatal in up to onethird of patients. ${ }^{9}$ Sternal-elevation techniques may facilitate safe mediastinal passage (ie, minimize pericardial trauma and risk of cardiac injury) by expanding the retrosternal space and improving thoracoscopic exposure.

A recent review by Haecker and colleagues ${ }^{10}$ found no (near)-fatal events among 4536 patients undergoing the Nuss procedure with sternal elevation. Therefore, the routine use of sternal-elevation techniques was recommended. ${ }^{10}$

Among the various methods for intraoperative elevation of the sternum, the crane technique is most frequently employed. ${ }^{10}$ This method uses a trans-sternal wire suture attached to an external table-mounted retractor device to generate sternal lift. Although the crane technique is regarded as effective, ${ }^{11,12}$ objective parameters such as maximum elevation or the lift required to allow a safe turn of the bar are missing in the literature, as underlined by Haecker and colleagues. ${ }^{10}$ In addition, it is unknown whether patients with a varying severity of pectus excavatum equally benefit from sternal elevation. The aim of this study was to evaluate the quantitative effect of the crane technique using intraoperatively acquired 3-dimensional (3D) images of the chest.

\section{METHODS \\ Study Design}

A single-center, prospective cohort study was conducted at Zuyderland Medical Centre (Heerlen, The Netherlands). Patients acted as their own control. The study was approved by the local research and ethics committee (METCZ; ID: 20200071; approval date: April 14, 2020) and subsequently submitted to the Clinicaltrials.gov registry (ID: NCT04418583; approval date: May 20,2020). Written informed consent was obtained before inclusion. The report was written in compliance with the Strengthening the Reporting of Observational Studies in Epidemiology statement. ${ }^{13}$

\section{Patients}

All consecutive patients with pectus excavatum undergoing surgical treatment by the Nuss procedure at our clinic (Zuyderland Medical Center, Heerlen, The Netherlands) were eligible for inclusion. Those with previous thoracic surgery were excluded.

\section{Crane Technique}

The crane technique has previously been described by Park and colleagues. ${ }^{11}$ In short, a surgical stainless-steel wire suture (Surgical Steel; Ethicon, Johnson \& Johnson, New Brunswick, NJ) is transversally passed through the anterior sternal cortex at the deepest point of the pectus (Figure 1, $A$ and $B$ ). Suture placement is performed under thoracoscopic guidance. In addition, careful interpretation of tactile feedback is used to envision the needle's trajectory and detect deviations. The Thompson rail clamp with unilateral crossbar, microadjustable clip-on handle, and rake blade (Thompson Surgical Instruments, Traverse City, Mich) is mounted to the operating table. The suture is attached to the Thompson retractor system to lift the suture along with the depressed sternum (Figure 1, $C$ ). To prevent the wire suture from coming loose during elevation, the ligated portion is bent and twisted along the suture itself (Figure 1, $C$ ). In addition, bending of the steel wire was minimized and the wire itself was not grasped with any surgical instrument to prevent introduction of weak spots. The anterior chest wall was lifted until the point at which maximum correction of the pectus deformity was achieved. (Figure $1, D$, and Video 1). In patients


FIGURE 1. Intraoperative view of the crane technique. A and B, A wire suture is passed through the anterior sternal cortex at the point of most severe depression. C, The wire suture is attached to a table-mounted retractor system, following which (D) sternal elevation is achieved. 


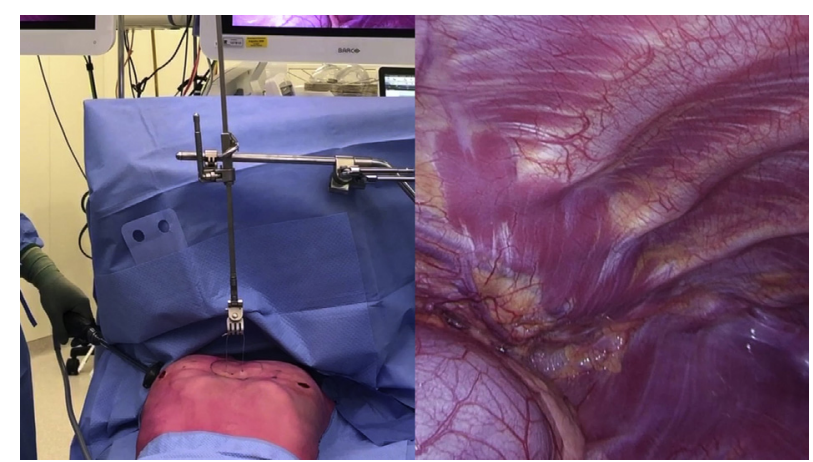

VIDEO 1. Intraoperative view of sternal elevation by the crane technique. Video available at: https://www.jtcvs.org/article/S2666-2507(21)00480-6/ fulltext.

for whom this was not possible, lift was continued until no additional anterior lift was visually obtained while winding the microadjustable handle of the crane system.

Following sternal elevation, the Nuss procedure is continued as described by Pilegaard. ${ }^{14}$ The retractor is dismantled after correct bar placement, including the assembly of 1 or more stabilizers. No procedural adjustments (regarding the Nuss procedure itself or bar configuration) were made after the routine introduction of the crane in early 2020 compared with the preceding period.

\section{Measurements and Variables}

Baseline patient characteristics, including sex, age, body mass index (BMI), preoperative Haller index, and preoperative complaints, were obtained from the electronic patient records. Three 3D images were acquired at different time points during the Nuss procedure, namely: (1) before skin incision, (2) following application of the crane and sternal lift, and (3) after Nuss bar placement and dismantling of the retractor. Before acquisition, line markings were made on the sterile drape and skin interface for image alignment purposes. All images were acquired by the Artec Leo (Artec3D, Luxembourg City, Luxembourg) imaging system. This is a handheld device that digitizes real-world objects using structured light and triangulation. Images were obtained by moving the imaging device around the anterior chest wall during apnea to avoid the introduction of motion artifacts. An anterior chest wall image is obtained in less than 6 seconds. ${ }^{15}$ Raw images were processed using Artec Studio 14.0 (Artec3D) in conjunction with the processing protocol described elsewhere. ${ }^{16}$

The force exerted by the crane simultaneously lifts the anterior chest wall and reduces the sternal depression, as shown in Figure 2, $A$ and $B$. This simultaneous effect was denoted as the combined elevation as reflected by the green line in Figure 2, $A$. The external depth was calculated in the sagittal direction with respect to a craniocaudal tangent of the anterior chest wall (Figure 3) by an automatic algorithm developed in MATLAB (version R2019a, The MathWorks Inc, Natick, Mass). ${ }^{17}$ Sagittal measurements were chosen over transversal measurements since the latter may overestimate depth in females due to the breasts. The pectus reduction was quantified by subtraction of the $3 \mathrm{D}$ image-derived external pectus excavatum depth, just before incision and following crane application (Figure 2, B).

Lift of the anterior chest wall was defined as the crane's combined elevation (in other words, simultaneous lift of the entire anterior chest wall and decrease of sternal depression; Figure 2, A) minus the pectus excavatum reduction. To determine the combined elevation, 3D images just before incision (Figure $4, A$ ) were compared with those made after crane application (Figure 4, B). Following rigid image alignment by manual selection of the line markings (Figure 4, $C$ and $D$ ), the maximum distance (at the point of deepest excavation) between the corresponding image points was retrieved by Artec Studio 14.0 (Artec3D). Color-coded surface distance maps were used to visualize differences in $\mathrm{mm}$ relative to the preoperative situation (Figure 4, E).

To compare the effect of the crane with the final result after definitive correction, the 3D images before incision were compared with the $3 \mathrm{D}$ images after Nuss bar implantation. Similarly, the maximum distance as well as the mean of signed distances were determined. These respectively depicted the maximum combined elevation at the deepest point

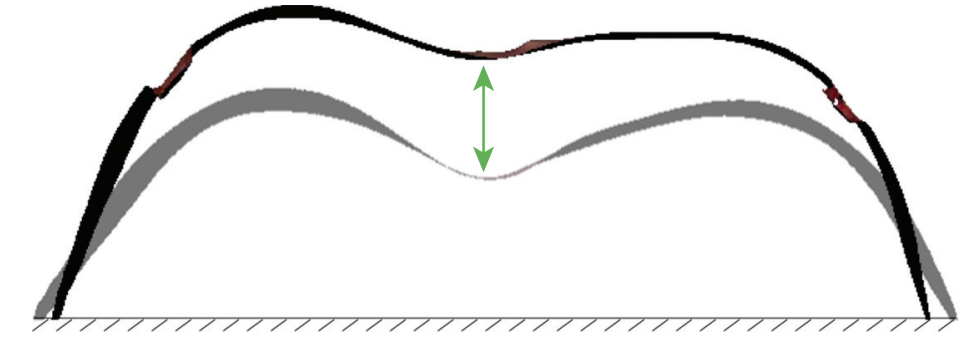

A

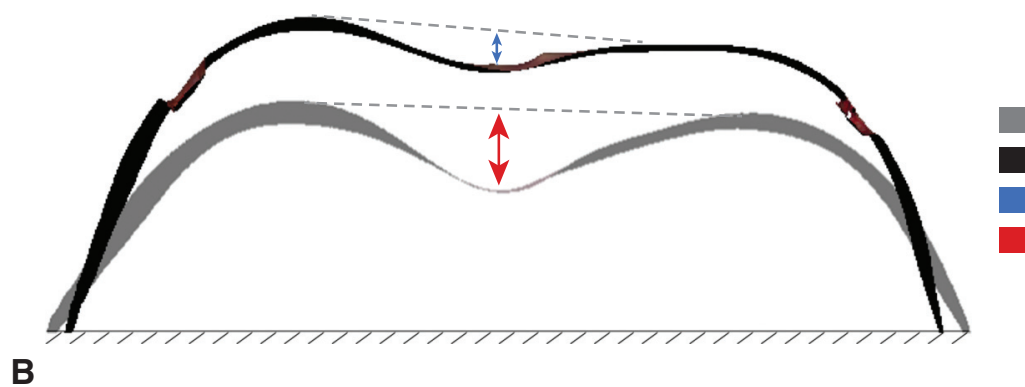

3D image slice prior to incision (transverse slice) 3D image after crane application (transverse slice) Combined elevation of the crane: simultaneous lift of the anterior chest wall and pectus excavatum reduction

FIGURE 2. Aligned transverse slice of the 3-dimensional (3D) image before incision and after crane application, demonstrating (A) the combined elevation achieved by the crane (ie, simultaneous lift of the anterior chest wall and pectus excavatum reduction), and (B) the reduction of the external pectus excavatum depth by the crane. Of note: subfigure B is merely for visual explanation, since the pectus reduction is measured in the sagittal direction. 


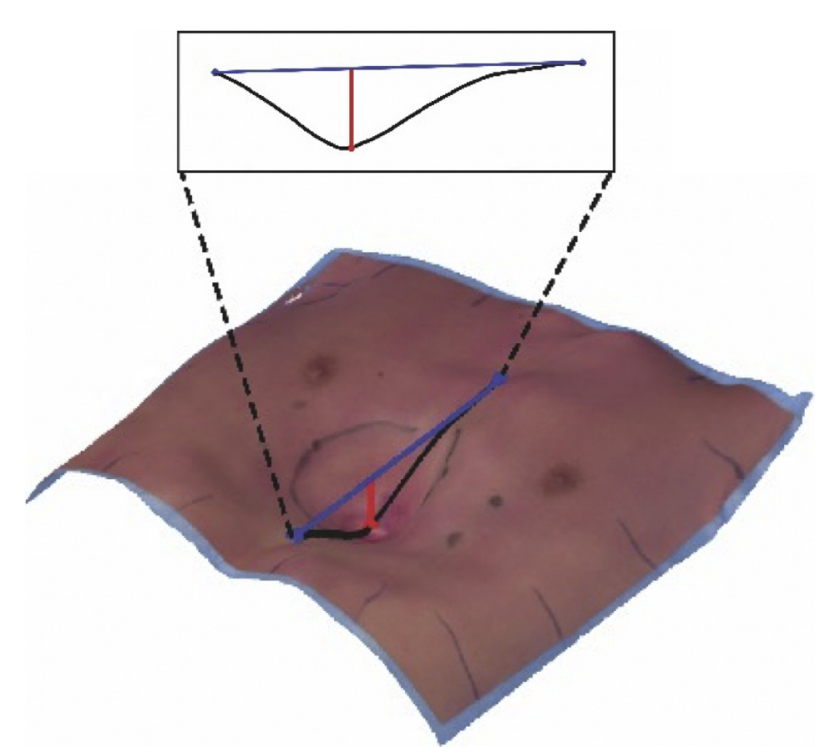

FIGURE 3. Measurement method of the 3-dimensional image-derived external depth, calculated in the sagittal direction with respect to a craniocaudal tangent of the anterior chest wall.

and average lift of the entire chest wall facilitated by the crane and Nuss bar.

\section{Statistical Analysis}

All statistical analyses were performed by SPSS statistics (IBM SPSS Statistics for MacOS, Version 27.0; IBM Corp, Armonk, NY). Continuous variables were reported as mean and standard deviation. In the presence of skewness, the median, interquartile range (IQR), and range were used. Nominal variables were reported as frequency and percentage.
Reduction of the external pectus excavatum depth by the crane technique was expressed as percentage. The relationship between the external depth and reduction was assessed by Pearson's or Spearman's rank correlation coefficient. In addition, the relationship between the preoperative Haller index and reduction was evaluated.

To compare the maximum and average combined elevation of the entire anterior chest wall caused by the crane and Nuss bar, measurements were submitted to the paired samples $t$-test or Wilcoxon signed rank test in the presence of skewness.

Given that no previous quantitative data regarding the effect of the crane technique was available, no sample size estimation could be performed; therefore, a sample size of 30 was chosen as a reasonable estimate.

\section{RESULTS}

Between May 2020 and December 2020, 31 patients underwent the Nuss procedure with sternal elevation by the crane technique, of whom 30 were included. One patient was excluded due to sterility issues of the retractor system. Ninety percent were male, with a median age of 15.5 years (IQR, 14.5-17.4; range, 13.2-26.9) and mean BMI of $19.0 \mathrm{~kg} / \mathrm{m}^{2}$ (standard deviation, 2.1). The median preoperative Haller index was 3.56 (IQR, 3.09-4.65; range, 2.38-10.21). Preoperative symptoms predominantly encompassed exercise intolerance $(57 \%)$ and body image disturbances $(67 \%)$.

The median preoperative external pectus excavatum depth was $18 \mathrm{~mm}$ (IQR, 11-23; range, 9-42). After sternal elevation by the crane, the external depth was reduced to $3 \mathrm{~mm}$ (IQR, 0-7; range, 0-18), indicating a reduction of $52 \%$ to $100 \%$ (median, $78 \%$; IQR, 63-100) in all patients.
A

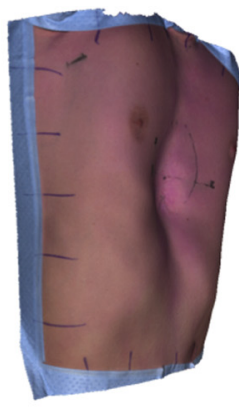

B

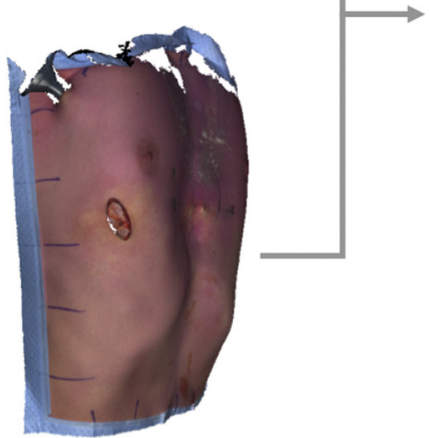

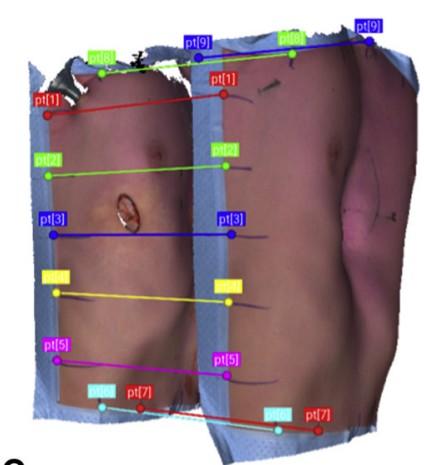

C

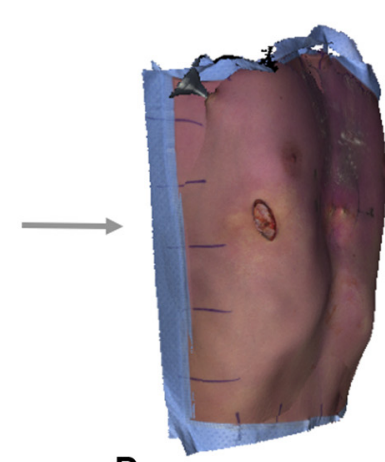

D



E

FIGURE 4. Method of 3-dimensional image alignment and creation of surface distance maps. A, Three-dimensional image acquired before skin incision and $\mathrm{B}$, after crane application. $\mathrm{C}$ and $\mathrm{D}$, Rigid alignment by manual section of the line markings. E, Resulting surface distance map, demonstrating the chest wall elevation after crane application relative to the preoperative image. 
In addition, a negative relationship was observed between the preoperative external depth and reduction achieved by the crane, characterized by a statistically significant Spearman's correlation coefficient of $-0.86(P<.001$; Figure 5, $A)$. In other words, the percentage of pectus reduction by the crane decreases with increasing pectus depth. Likewise, the preoperative external depth demonstrated a correlation of $0.85(P<.001$; Figure $5, B)$ with the absolute reduction in millimeters. No significant correlation was found between the Haller index and reduction achieved by the crane (Spearman's correlation coefficient: $-0.35 ; P=.061$ ).

Comparison of 3D images acquired just before incision and after crane application revealed a median maximum combined elevation of the anterior thoracic wall (at the point of most severe depression, indicated by the green arrow in Figure 2, $A$ ) of 39 mm (IQR, 33-48; range, 25-115). Besides the aforementioned reduction in external pectus depth, the crane thus provides a maximum elevation of the anterior chest wall of $26 \mathrm{~mm}$ (IQR, 19-32; range, 6-91).

The Nuss bar achieved a greater combined elevation than the crane at the deepest point of the pectus (median, $44 \mathrm{~mm}$; IQR, 37-58; range, 26-93 vs crane median, $39 \mathrm{~mm}$; IQR, 3348 ; range, $25-115 ; P=.021)$. In contrast, the crane facilitated a greater average elevation of the entire thoracic wall (median, $9 \mathrm{~mm}$; IQR, 6-11; range, 1-14 vs bar median: $4 \mathrm{~mm}$; IQR, 3-6; range, 0.4-12; $P<.001)$. Thus, the crane facilitates a more diffuse lift of the entire anterior chest compared to the final bar. This was visually confirmed by the surface distance maps in Figure 6.

The median duration of surgery, from skin incision to closure, was 34 minutes (IQR, 27-43; range, 22-75). No intraoperative or postoperative crane related complications occurred. The key findings have been emphasized in Figure 7.

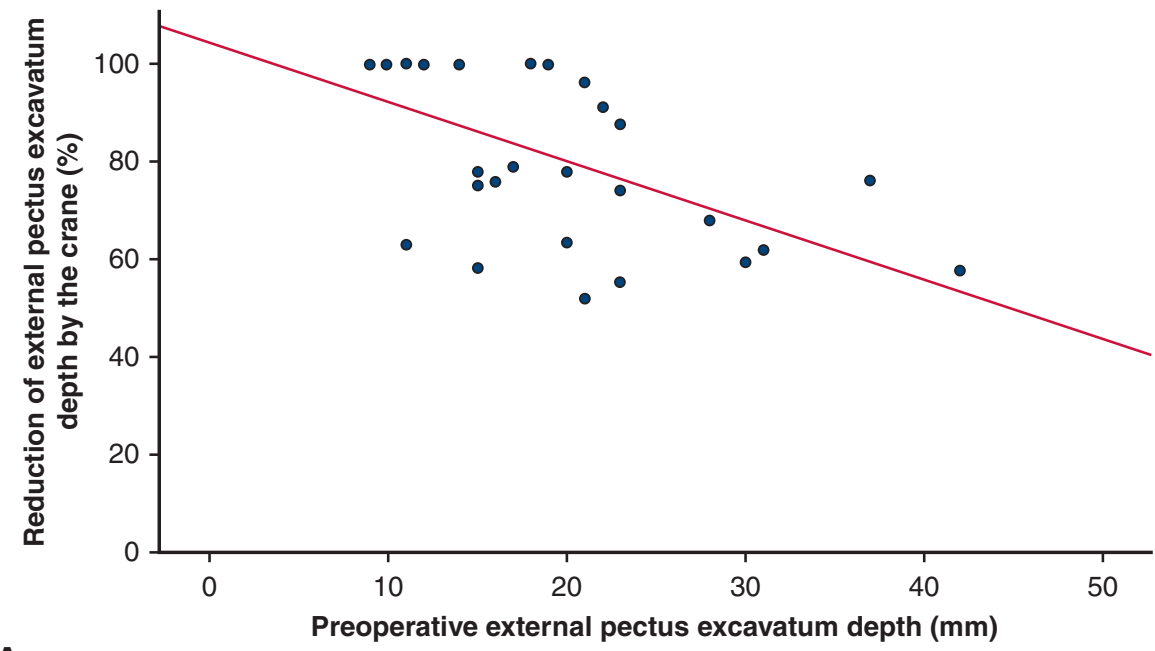

A

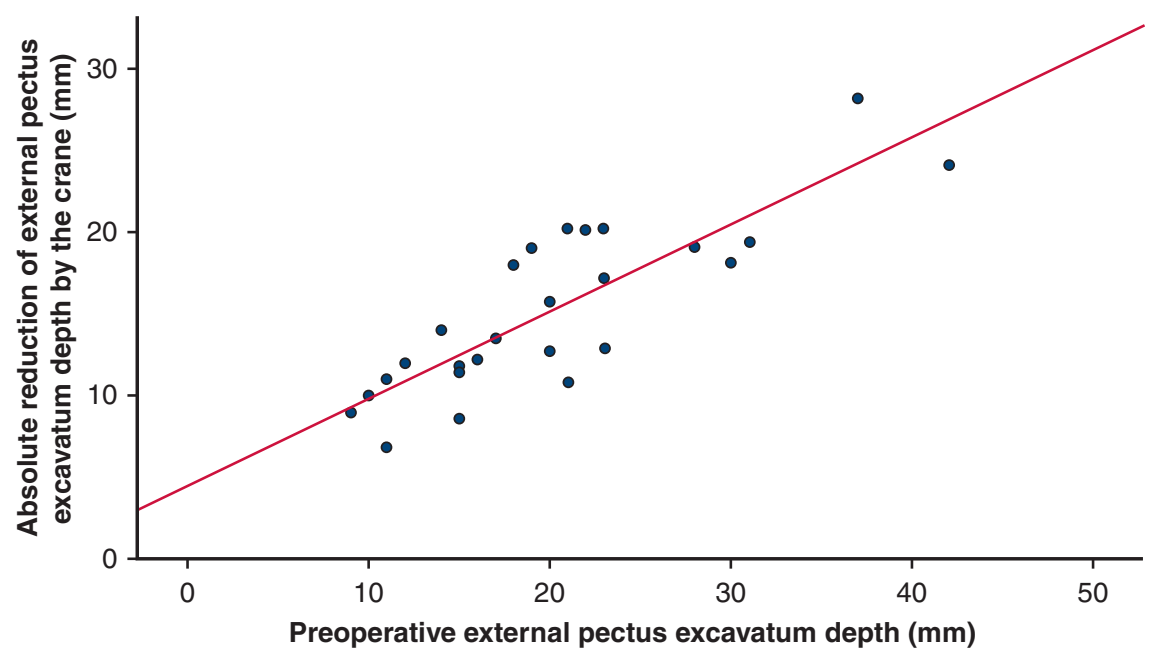

FIGURE 5. Two scatter plots with corresponding linear regression lines demonstrating (A) the percentual reduction of the external pectus excavatum depth and (B) the absolute reduction of the external pectus excavatum depth by the crane technique versus the preoperative external pectus excavatum depth. 


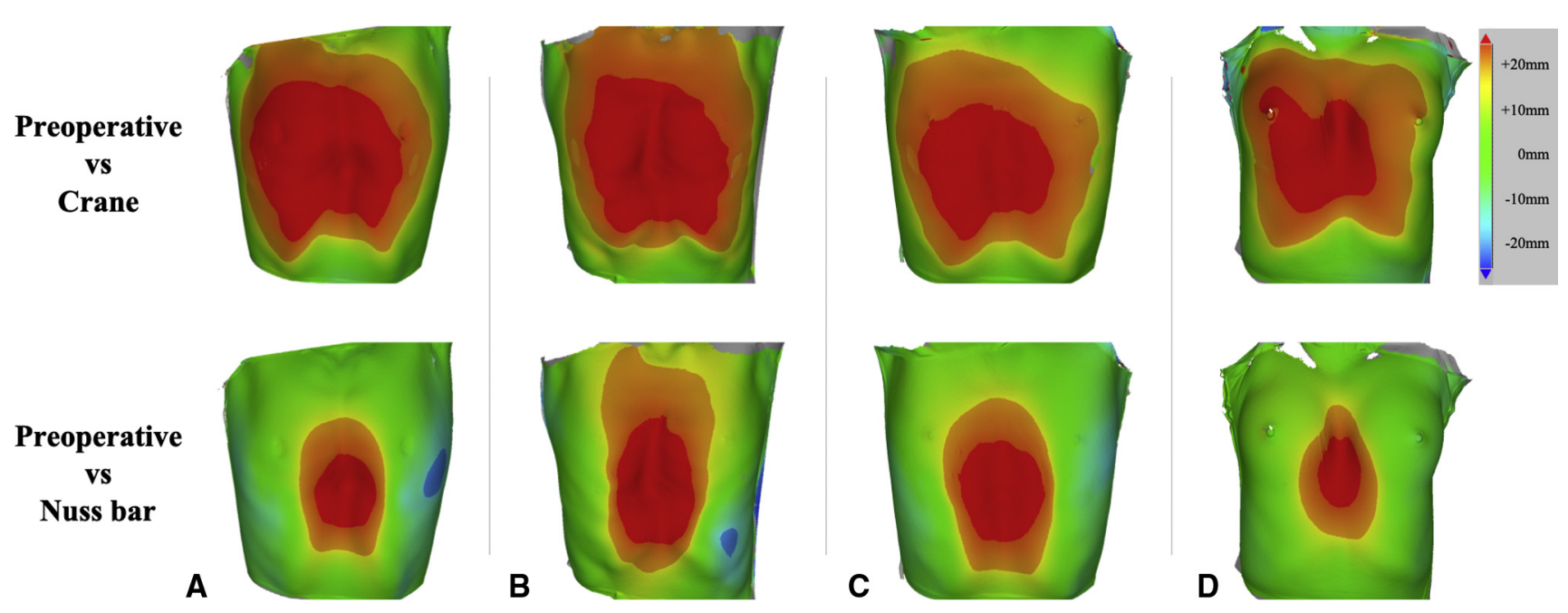

FIGURE 6. Color-coded surface distance maps (in millimeters) of (A-D) 4 distinct patients. The top row reveals the differences between the preoperative situation and after crane application, whereas the bottom row reveals the differences between the preoperative situation and after Nuss bar implantation.

\section{DISCUSSION}

The current study is the first to objectively assess the quantitative effect of the crane technique on the anterior thoracic wall using intraoperatively acquired 3D images of the chest. Sternal lift by the crane technique was found to facilitate a median pectus depth reduction of $78 \%$, ranging from $52 \%$ to $100 \%$ per individual patient and diminishing with increasing pectus depth. This corresponded with a median reduction of $13 \mathrm{~mm}$ (IQR, 11-19), while at the same time, a maximum anterior chest wall elevation of $26 \mathrm{~mm}$ (IQR, 19-32) was achieved. This greater elevation of the chest wall compared with reduction of the deformity at its deepest point is likely to be caused by the greater rigidity of the deformity (ie, sternum and adjacent costal cartilage) in comparison with the remainder of the thoracic wall.

When we compared the combined chest wall elevation by the crane to that achieved by the final bar, the former was observed to affect a significantly larger surface of the thoracic wall $(P<.001$; Figure 6$)$. This difference is explained by the forces applied. The trans-sternal steel wire in conjunction with the Thompson retractor produced a unidirectional upward force, counteracted by the elasticity of the chest wall and gravitational forces of the body weight (Figure $8, A$ ). The same chest wall elasticity applies to the Nuss bar, although the bar itself also exerts inwardly directed forces at the hinge points (Figure 8, $B$ ) and the position of the stabilizers causing relative bilateral chest wall depressions, as indicated by the blue areas in Figure 6, A and $C$.

Despite the fact that the crane technique facilitates a more diffuse lift than the implanted Nuss bar, the bar generates a significantly greater combined lift at the deepest point of the pectus $(P=.021)$. This is the result from the bars' convex shape to achieve an anatomical position of the sternum and adjacent costal cartilage over time. ${ }^{14}$ In contrast, the goal of the crane is solely to provide a temporary elevation of the sternum for safe retrosternal passage.

A correlation of -0.86 was shown between the reduction of the external pectus excavatum depth by the crane and the preoperative external pectus excavatum depth. Yet, no meaningful correlation was observed with the Haller index $(-0.35 ; P=.061)$. This may be because the Haller index is affected by the thoracic shape (eg, barrel vs flat-shaped).

Although the routine use of sternal elevation during the Nuss procedure is recommended by Haecker and colleagues, ${ }^{10}$ elevation is potentially more beneficial in deep deformities in terms of risk reduction of serious adverse events. In the present study, we found the relative elevation to decrease with increasing pectus excavatum depth. Nevertheless, a minimum reduction of $52 \%$ was observed. Even for the 4 patients with a Haller index larger than 7.00 (up to 10.21). Thus, despite the relatively lower percentual reduction in deep deformities, sternal elevation by the crane technique is advocated given that even a minimum reduction of $52 \%$ can drastically increase the retrosternal space which facilitates safer mediastinal passage (as observed in Figure 5, $B$ ), but also reduces rotational forces during flipping (rotation during placement) of the Nuss bar. It thus also facilitates minimally invasive repair by the Nuss procedure in cases with extreme sternal depression, which would otherwise have undergone a modified Ravitch procedure.

Besides the crane technique, various other methods exist to achieve sternal elevation. Among them is the vacuum bell. This cup-shaped device generates lift through subatmospheric pressure and does not require skin incision or puncture. However, the vacuum bell can cause hematoma and is only described to be effective in patients with low BMI and shallow deformities. ${ }^{18}$ Moreover, in European countries, there is a trend toward using shorter bars, ${ }^{14}$ requiring more medially placed incisions, complicating 




3D: Three-dimensional

FIGURE 7. Key findings regarding the quantitative analysis of the crane technique, used for sternal elevation during the Nuss procedure. The aim was to evaluate the quantitative effect of the crane technique. Using 3-dimensional $(3 D)$ images, we observed a median $78 \%$ reduction of the external pectus depth among 30 patients, although its effect was observed to lessen with increasing depth.

vacuum bell use. One may also manually lift the sternum, although this is more invasive, as it requires an additional subxiphoidal skin incision as well as elevation or removal of the xiphoid process. ${ }^{19,20}$ The same effect can be achieved by a substernal spade-like device attached to a stabilizer system following xiphoidectomy. ${ }^{21}$

Less-invasive techniques include the use of a percutaneous sternal bone clamp with retractor system ${ }^{22}$ or a handheld horseshoe-shaped sternal elevator, which is inserted through the standard anterolateral incision. ${ }^{23}$ Similarly, one can use 2 Langenbeck retractors to manually lift the chest wall without additional surgical skin trauma. ${ }^{24}$ The crane technique, used in the current series, has the advantage of minimal surgical trauma due to the use of steel wire requiring only 2 skin punctures and the use of a retractor system by which continuous and steady elevation is ensured. In addition, the crane method does not interfere with flipping of the Nuss bar, in contrast to, for example, the technique using the Langenbeck retractors. Nevertheless, given the variety of sternal elevation techniques, future studies should aim to compare and determine the optimal technique based on the trade-off between efficacy and additional surgical trauma. The optimal technique may differ among patient groups. For example, different techniques may be more effective for older compared with young patients due to a greater chest wall rigidity, enlarging the forces needed to obtain sternal elevation. ${ }^{25}$ The same may apply for cup-shaped versus saucer-shaped deformities, but also for redo compared with primary surgery.

Although the crane technique has no absolute contraindications, the presence of a large amount of presternal subcutaneous fat tissue may challenge wire suture placement and thus pose a relative contraindication. However, given that pectus patients are predominantly slender with limited amounts of subcutaneous tissue, this is rather rare than common. In addition, sternal tilt could complicate suture placement in severe cases. In such cases, as well as in patients with obesity, longitudinal placement may be attempted instead of the usual transverse placement (Figure 1,C).

As mentioned in the Methods section, several failsafe mechanisms were incorporated to deal with 


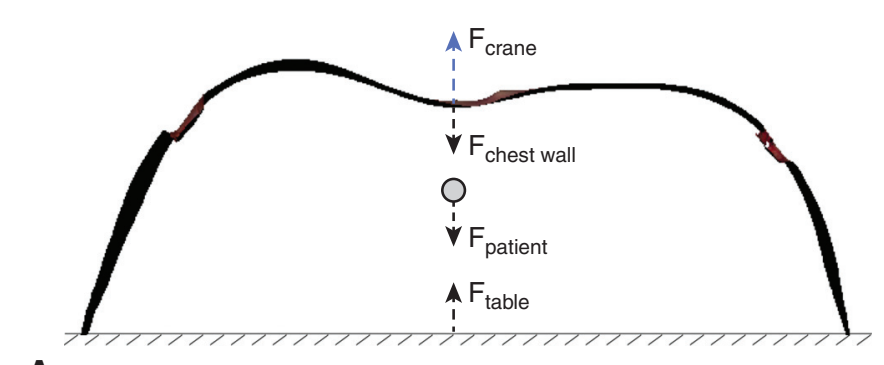

A

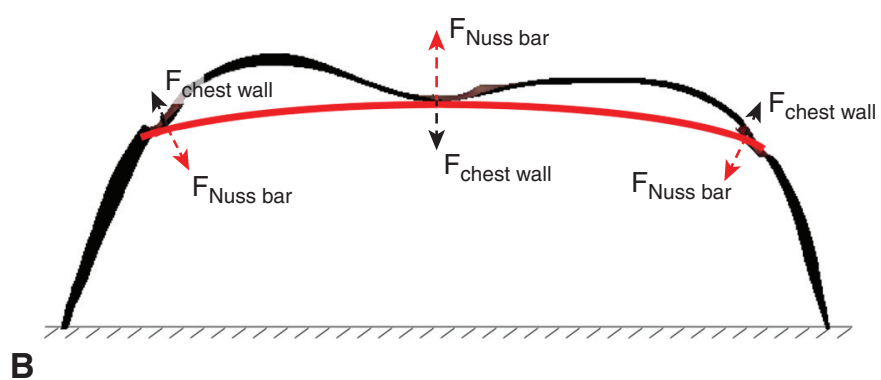

FIGURE 8. A simplified representation of the direction of forces being exerted during (A) sternal elevation by the crane technique and (B) definitive correction by the metal Nuss bar, which is depicted in red. Of note: this is a simplified representation wherein the vector's length does not correlate with the amount of force being exerted.

potential weaknesses of the crane technique. Employing these mechanisms, we observed no crane related complications in the present study (eg, cardiac puncture during suture placement). Neither did Haecker and colleagues $^{10}$ find any cardiac complications among 3047 patients who underwent the Nuss procedure with sternal elevation by the crane technique. In other words, the risk of cardiac injury during transsternal suture placement is negligible.

A limitation of the crane technique is that it requires additional actions during surgery, as well as additional costs of the surgical steel wires and a one-time investment in a retractor system. However, the operation time in the current series (34 minutes; IQR, 27-43) compared with a published retrospective study of our group including 222 patients (median: 30 minutes; IQR, 25-39) with comparable Haller indices (mean: 3.9 compared with a median of 3.56 in the current series) from the same center yielded a median difference of only 4 minutes. ${ }^{26}$ This minor increase may be deemed negligible, given the potential benefits of the crane technique.

The validity of measurements is a direct derivative of the validity of the imaging system employed. In the current series, all 3D images were acquired by the handheld Artec Leo, which has previously been shown to be accurate and reproducible for acquisition of anterior chest wall images. ${ }^{15}$ However, errors could theoretically still be introduced by the semimanual alignment of images.

We did not measure the upward force delivered by the crane. This may also be regarded as limitation. As previously mentioned, a variable amount of force was applied (ie, the anterior chest wall lift was continued until the sternal part of the deformity was fully reduced or until no additional anterior lift was visually obtained while winding the microadjustable handle) to maximize expansion of the retrosternal space. Given that the crane's goal is to expand the retrosternal space to allow safe mediastinal passage in conjunction with the fact that the required forces to achieve sternal lift increase with age $\mathrm{e}^{25,27}$ and severity (Haller index $>5.0),{ }^{27}$ our aim was not to evaluate the amount of sternal lift at a certain force but to evaluate the achievable amount of lift. Moreover, in clinical practice, a pectus surgeon will not halt lifting at a certain attained force during surgery while an even greater lift with potentially safer mediastinal passage can be achieved.

The sum of forces (Figure 8, A) that determines lift also encompasses the strength of the retractor system used. In the current study, we used a retractor with unilateral rail clamp. A system with bilateral rail clamps and crossbar may theoretically be more rigid and thus provide a greater elevation under the assumption that an even greater elevation is achievable without cutting out of the steel wire through the sternal cortex. In theory, the amount of upward force produced by a retractor system may even lift the entire torso of the operating table and affect measurements. Nevertheless, in the current series, we matched images based on the line markings and thus only considered relative changes of the anterior chest wall.

Evaluating the percentual reduction achieved by the crane technique, linear measurements were used. Yet, given the variety of pectus presentations (eg, symmetric vs 
asymmetric), volumetric differences may have been more informative. However, we deliberately refrained from the use of volumetric changes, since the excavation in pectus excavatum has no strict objectifiable borders, which become even more vague after lift by the crane technique.

The presumed clinical benefits primarily relate to improved safety through decreased risk of injury to the heart and great vessels and diminished risk for scraping of the intrathoracic anterior chest wall where the internal mammary arteries are at risk for injury. Moreover, reduction of rotational forces during flipping of the Nuss bar that may prevent stripping of the intercostal muscles.

The primary aim of sternal elevations techniques is to facilitate safe mediastinal passage (ie, minimize pericardial trauma and risk of cardiac injury) by expanding the retrosternal space and improving thoracoscopic exposure. In contrast, it potentially also broadens the eligibility criteria for the Nuss procedure to more severe and complex cases.

Our results demonstrate the efficacy of the crane technique on intraoperative elevation of the sternum of patients having surgery for pectus excavatum by the Nuss bar technique. Whether this technique also translates into benefits for clinical results warrants further investigation.

\section{CONCLUSIONS}

The crane provides an effective technique for sternal elevation during the Nuss procedure, facilitating a $78 \%$ reduction of the external pectus excavatum depth, although its effect lessens with increasing depth. Besides a temporary reduction of the deformity, the crane causes a diffuse elevation of the entire anterior chest wall.

\section{Conflict of Interest Statement}

The authors reported no conflicts of interest.

The Journal policy requires editors and reviewers to disclose conflicts of interest and to decline handling or reviewing manuscripts for which they may have a conflict of interest. The editors and reviewers of this article have no conflicts of interest.

\section{References}

1. Chung CS, Myrianthopoulos NC. Factors affecting risks of congenital malformations. I. Analysis of epidemiologic factors in congenital malformations. Report from the Collaborative Perinatal Project. Birth Defects Orig Artic Ser. 1975; 11:1-22.

2. Fokin AA, Steuerwald NM, Ahrens WA, Allen KA. Anatomical, histologic, and genetic characteristics of congenital chest wall deformities. Semin Thorac Cardiovasc Surg. 2009;21:44-57.

3. Kowalewski J, Brocki M, Dryjanski T, Zolyński K, Koktysz R. Pectus excavatum: increase of right ventricular systolic, diastolic, and stroke volumes after surgical repair. J Thorac Cardiovasc Surg. 1999;118:87-92.

4. Gürkan U, Aydemir B, Aksoy S, Akgöz H, Tosu AR, Öz D, et al. Echocardiographic assessment of right ventricular function before and after surgery in patients with pectus excavatum and right ventricular compression. Thorac Cardiovasc Surg. 2014;62:231-5.
5. Oezcan S, Attenhofer Jost CH, Pfyffer M, Kellenberger C, Jenni R, Binggeli C, et al. Pectus excavatum: echocardiography and cardiac MRI reveal frequent pericardial effusion and right-sided heart anomalies. Eur Heart J. 2012;13:673-9.

6. Malek MH, Berger DE, Housh TJ, Marelich WD, Coburn JW, Beck TW. Cardiovascular function following surgical repair of pectus excavatum. Chest. 2006; 130:506-16.

7. Mao YZ, Tang S, Li S. Comparison of the Nuss versus Ravitch procedure for pectus excavatum repair: an updated meta-analysis. J Pediatr Surg. 2017;52:1545-52.

8. Nuss D, Kelly RE, Croitoru DP, Katz ME. A 10-year review of a minimally invasive technique for the correction of pectus excavatum. J Pediatr Surg. 1998;33:545-52.

9. Hebra A, Kelly RE, Ferro MM, Yüksel M, Campos JRM, Nuss D. Life-threatening complications and mortality of minimally invasive pectus surgery. $J$ Pediatr Surg. 2018;53:728-32.

10. Haecker FM, Krebs T, Kocher GJ, Schmid RA, Sesia SB. Sternal elevation techniques during the minimally invasive repair of pectus excavatum. Interact Cardiovasc Thorac Surg. 2019;29:497-502.

11. Park HJ, Jeong JY, Jo WM, Shin JS, Lee IS, Kim KT, et al. Minimally invasive repair of pectus excavatum: a novel morphology-tailored, patient-specific approach. $J$ Thorac Cardiovasc Surg. 2010;139:379-86.

12. Jeong JY, Lee J. Use of needlescope and crane technique to avoid cardiac injury in Nuss procedure. Ann Thorac Surg. 2014;98:386-7.

13. von Elm E, Altman DG, Egger M, Pocock SJ, Gøtzsche PC, Vandenbroucke JP. The strengthening the reporting of observational studies in Epidemiology (STROBE) statement: guidelines for reporting observational studies. Lancet. 2007;370:1453-7.

14. Pilegaard HK. Short Nuss bar procedure. Ann Cardiothorac Surg. 2016;5:513-8

15. Daemen JHT, Loonen TGJ, Verhulst AC, Maal TJJ, Maessen JG, Vissers YLJ, et al. Three-dimensional imaging of the chest wall: a comparison between three different imaging systems. J Surg Res. 2020;259:332-41.

16. Daemen JHT, Loonen TGJ, Coorens NA, Maessen JG, Maal TJJ, Hulsewé KWE, et al. Photographic documentation and severity quantification of pectus excavatum through three-dimensional optical surface imaging. J Vis Commun Med. 2020;43:190-7.

17. Coorens NA, Daemen JHT, Slump CH, Loonen TGJ, Vissers YLJ Hulsewé KWE, et al. The automatic quantification of morphological features of pectus excavatum based on three-dimensional images. Semin Thorac Cardiovasc Surg. 2021. In press.

18. Togoro SY, Tedde ML, Eisinger RS, Okumura EM, de Campos JRM, PêgoFernandes PM. The Vacuum Bell device as a sternal lifter: an immediate effect even with a short time use. J Pediatr Surg. 2018;53:406-10.

19. Gould JL, Sharp RJ, Peter SD, Snyder CL, Juang D, Aguayo P, et al. The minimally invasive repair of pectus excavatum using a subxiphoid incision. Eur J Pediatr Surg. 2017;27:2-6.

20. St Peter SD, Sharp SW, Ostlie DJ, Snyder CL, Holcomb GW III, Sharp RJ. Use of a subxiphoid incision for pectus bar placement in the repair of pectus excavatum. J Pediatr Surg. 2010;45:1361-4.

21. Johnson WR, Fedor D, Singhal S. A novel approach to eliminate cardiac perforation in the nuss procedure. Ann Thorac Surg. 2013;95:1109-11.

22. Jaroszewski DE, Johnson K, McMahon L, Notrica D. Sternal elevation before passing bars: a technique for improving visualization and facilitating minimally invasive pectus excavatum repair in adult patients. J Thorac Cardiovasc Surg. 2014; 147:1093-5.

23. Takagi S, Oyama T, Tomokazu N, Kinoshita K, Makino T, Ohjimi H. A new sternum elevator reduces severe complications during minimally invasive repair of the pectus excavatum. Pediatr Surg Int. 2012;28:623-6.

24. Tedde ML, de Campos JR, Wihlm JM, Jatene FB. The Nuss procedure made safer: an effective and simple sternal elevation manoeuvre. Eur J Cardiothorac Surg. 2012;42:890-1.

25. Weber PG, Huemmer HP, Reingruber B. Forces to be overcome in correction of pectus excavatum. J Thorac Cardiovasc Surg. 2006;132:1369-73.

26. de Loos ER, Daemen JHT, Pennings AJ, Heuts S, Maessen JG, Hulsewé KWE, et al. Minimally invasive repair of pectus excavatum by the Nuss procedure: the learning curve. J Thorac Cardiovasc Surg. December 10, 2020 [Epub ahead of print].

27. Fonkalsrud EW, Reemtsen B. Force required to elevate the sternum of pectus excavatum patients. J Am Coll Surg. 2002;195:575-7.

Key Words: crane technique, sternal elevation, Nuss procedure, pectus excavatum 